Hand- oder Fußekzem und weitere neun andere Hautsymptome. Das am häufigsten nachgewiesene Kontaktallergen war Nickel (43\%) - ähnlich wie auch bei Erwachsenen - gefolgt von Kaliumdichromat, Duftstoffmix und Thiomersal. Die Pflegesubstanzen riefen nur selten ein positives Ergebnis hervor. Bei sechs Kindern konnten Substanzen aus ihren Schuhen, bei fünf Stoffe aus den Schienbeinschützern als allergieauslösend identifiziert werden.

Kommentar: Kontaktallergien müssen bei Kindern mit Ekzemen immer differenzialdiagnostisch erwogen werden. Zur Abklärung sind entsprechende Tests auch bei jüngeren Kindern sinnvoll. Die vorliegende holländische Studie konnte im- merhin fünf Kinder im Vorschulalter mit einem oder mehreren positiven Testergebnissen ermitteln. Dr. Ulrich Mutschler

Waard-van der Spek FB de et al. Patch tests in children with suspected allergic contact dermatitis: a prospective study and review of the literature. Dermatology 2009; 218: 119-125

\title{
Plötzlicher Kindstod: enges Wickeln nützlich oder schädlich?
}

\section{In den USA und in Australien erfreut sich das enge Wickeln von Kindern zunehmender Beliebtheit. Welche neurophysiologischen Effekte diese Methode hat, wurde in einer kleinen kontrollierten Studie untersucht.}

ls mögliche Ursache für das Sudden Infant Death Syndrome (SIDS) gilt die Unfähigkeit, adäquate Arousal-Effekt (Weckreaktion) auf kardiovaskulären oder respiratorischen Stress zu entwickeln. Welchen Effekt enges Wickeln auf diese Reaktionen hat, wird kontrovers diskutiert.

In eine australische Studie wurden 27 gesunde Säuglinge aufgenommen. Alle waren termingerecht geboren und normalgewichtig. Die Mütter hatten während der Schwangerschaft nicht geraucht, alle Kinder wurden gestillt. Zum Einschlafen wurden die Kinder stets in Rücklage gebracht. 15 Säuglinge wurden regelmäßig eng gewickelt, bei den zwölf anderen erfolgte diese Maßnahme nicht.

Im Alter von drei bis vier Wochen und im Alter von drei Monaten erfolgte in einem Schlaflabor eine Polysomnografie. Um die Kinder auf ihre Fähigkeit zu Arousal-Reaktionen zu testen, erfolgten während des Schlafes für fünf Sekunden pulsartige Luftströme mit einer Frequenz von $3 \mathrm{~Hz}$ im Bereich der Nasenlöcher.

Im Alter von drei bis vier Wochen hatte das enge Wickeln keinen signifikanten Effekt auf die Arousal-Grenze (notwendiger Druck des Luftstroms für Auslösen eines Arousal-Effekts). Bei den drei Monate alten Säuglingen war nach engem Wickeln diese Grenze signifikant höher - dies galt aber nur für Kinder im tiefen Schlaf.

Das enge Wickeln zeigte außerdem keinen signifikanten Effekt auf die Fre- quenzen der subkortikalen oder kortikalen Aktivität - vorausgesetzt, es handelte sich um Kinder, die regelmäßig auf diese Art zu Bett gebracht wurden. Bei den Säuglingen, die normalerweise nicht eng gewickelt worden waren und bei denen nun erstmals diese Maßnahme erfolgte, waren höhere Luftstromdrücke notwendig, um einen Arousal-Effekt hervorzurufen. Die kortikale Aktivität war hier vermindert. Dies galt wiederum für drei Monate alte Kinder im tiefen Schlaf.

Kommentar: Es handelt sich um eine sehr aufwendig durchgeführte Studie, die sich mit der neuen Säuglingspflegetechnik des engen Wickelns beschäftigt. Dieses enge Wickeln wird zunehmend populär, da es doch wohl eine stark beruhigende Wirkung hat. Teilweise auch durch esoterische Konzepte, dem Motto entsprechend „das Kind braucht Halt“, wird diese Technik zurzeit mehr und mehr angewandt.

Diese Untersuchung aus Australien zeigt, dass die Beruhigung durch das enge Wickeln die Arousal-Reaktion reduziert - eigentlich naheliegend: Ruhe, längere Tiefschlafphasen und eine beruhigende Umgebung werden die Wirkung von Stressoren, die zu einer Arousal-Reaktion führen, zurückdrängen. Die Konsequenz, nämlich dass die Arousal-Reaktion, die eine große Rolle beim plötzliches Kindstod spielt, durch enges Wickeln reduziert wird, ist insofern nicht überraschend.

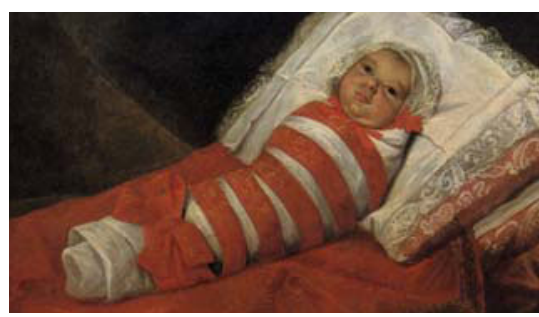

Enges Wickeln war schon mal in (hier das Werk „Mathias von Jenisch als Fatschenkind" aus dem 17. Jahrhundert) und kommt jetzt scheinbar wieder in Mode.

Der Erklärungsmechanismus eines ähnlichen Effektes beim Schlafen von Säuglingen im Zimmer der Eltern (dort ist es lauter), deuten in eine ähnliche Richtung. Dass mehr Ruhe, mehr Tiefschlaf und weniger Arousal das Risiko für Kindstod in einer Risikopopulation erhöhen können und die daraus zu ziehenden Konsequenzen, könnten Inhalt einer tiefphilosophischen Debatte sein.

Insgesamt verdeutlicht dieses Ergebnis wieder, dass neue Techniken zur Beruhigung und Pflege von Säuglingen - teilweise mit esoterischen, ideologischen Konzepten verbrämt - nicht zu euphorisch in die Säuglingspflege eingeführt werden sollen. Alles hat seinen Preis! Umgekehrt kann es Situationen geben, wie bei Schreikindern, wo in einer professionellen Risiko-Nutzen-Abwägung enges Wickeln sinnvoll sein kann [J Pediatr 2006; 149: 512-7]. Bewährte Strategien, Anleitungen und Gespräche mit Eltern, insbesondere auch bei der Behandlung von Schreibabys, die etablierte Kenntnisse über die Prävention des plötzlichen Kindstodes keineswegs ignorieren, werden immer der praktikablere Weg sein.

Prof. Dr. Jens Möller/af

Richardson HL et al. Minimizing the risks of sudden infant death syndrome: to swaddle or not to swaddle? J Pediatr 2009; 155: 475-81 